\title{
Acknowledgement to Reviewers of Medical Sciences in 2016
}

\author{
Medical Sciences Editorial Office \\ MDPI AG, St. Alban-Anlage 66, 4052 Basel, Switzerland; medsci@mdpi.com \\ Published: 13 January 2017
}

The editors of Medical Sciences would like to express their sincere gratitude to the following reviewers for assessing manuscripts in 2016.

We greatly appreciate the contribution of expert reviewers, which is crucial to the journal's editorial process. We aim to recognize reviewer contributions through several mechanisms, of which the annual publication of reviewer names is one. Reviewers receive a voucher entitling them to a discount on their next MDPI publication and can download a certificate of recognition directly from our submission system. Of course, in these intiatives we are careful not to compromise reviewer confidentiality. Many reviewers see their work as a voluntary and often unseen part of their role as researchers. We are grateful to the time reviewers donate to our journals and the contribution they make.

If you are interested in becoming a reviewer for Medical Sciences, see the link at the bottom of the webpage http://www.mdpi.com/reviewers.

The following reviewed for Medical Sciences in 2016:

Adler, Stuart P.

Ahmad, Ali

Armand-Lefèvre, Laurence

Azarnia Tehran, Domenico

Bartoloni, Alessandro

Biunno, Ida

Caceres, Alejandro

Casartelli, Luca

Chen, Chiung-Tong

Cimanga, Kanyanga

Cros, Gérard

D'Elia, Lanfranco

Di Tola, Marco

Dias, Luís G.

Djilali-Saiah, Idriss

Dollard, Sheila

Donnellan, Anne M.

Eckel, Stephen

Emond, Claude.

Francis, Joel

$\mathrm{Fu}$, Sherleen

Gilbert, Caroline

Govoni, Gregory R.

Greco, Luigi

Gupta, Aditi
Gutierrez, Claudio

Haglin, Lena

Hanson, Nancy D.

Hardy, Melinda

Isman, Murray

Jakubowska, Monika

Jampol, Lee

Jenkins, Jill A.

Joan, Smith-Sonneborn

Kanakkanthara, Arun

Kang, Yanyong

Kasarda, Don

Kashanchi, Fatah

Kasinski, Andrea

Kundu, Aishwarya

Kyvelidou, Anastasia

Lagasse, Blythe

Lakhkar, Anand

Lederkremer, Gerardo Z.

Liberti, Sascha Emilie

Mariussen, Espen

Mascaraque, Cristina

Matsumoto, Yasuhiko

Mcmorrow, Tara

Meijles, Daniel
Mulder, Sasja

Nana, Andre W.

Nath, Rajendra

Navarro, Ferran

Nenna, Raffaella

Osborne, Brenna

Paganetti, Paolo

Pani, Bibhusita

Peng, Chi Chung

Picarelli, Antonio

Porta, Sepp

Prater, M. Renee

Pusapati, Ganesh

Ravegnini, Gloria

Remelli, Maurizio

Rephaeli, Ada

Santulli, Gaetano

Sapone, Anna

Schauss, Alex

Schettino, Giuseppe

Shlipak, Michael G.

Sigafoos, Jeff

Siltberg-Liberles, Jessica

Simcox, Judith 

Teixeira Damasceno, Nágila
Raquel

Van Grevenynghe, Julien
Viggiano, Davide

Vinchi, Francesca

Wang, Kesheng
Yang, Xue

Zhang, Guozhi

Zong, Sheng Guo

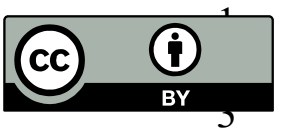

(C) 2017 by the authors. Submittedfor possible open access publication under the terms and conditions of the Creative Commons Attribution (CC-BY) license (http://creativecommons.org/licenses/by/4.0/). 